\title{
Pseudoprogression presenting as intestinal perforation in non-small cell lung cancer treated with anti-PD-1: A case report
}

\author{
HEE KYUNG KIM ${ }^{1}$, SEUNG-WOO BAEK ${ }^{1}$, YUSOOK JEONG ${ }^{1}$, \\ YAEWON YANG $^{1}$, JIHYUN KWON ${ }^{1}$, HYE SOOK HAN ${ }^{1,2}$, JIN-YOUNG AN ${ }^{2,3}$, \\ CHANG GOK WOO ${ }^{4}$, OK-JUN LEE ${ }^{2,4}$, TAEK GU LEE ${ }^{2,5}$ and KI HYEONG LEE ${ }^{1,2}$ \\ ${ }^{1}$ Division of Hematology and Oncology, Chungbuk National University Hospital; \\ ${ }^{2}$ Department of Internal Medicine, College of Medicine, Chungbuk National University; \\ ${ }^{3}$ Division of Pulmonology; Departments of ${ }^{4}$ Pathology and ${ }^{5}$ Surgery, Chungbuk National University Hospital, \\ Cheongju, North Chungcheong 28644, Republic of Korea
}

Received March 4, 2019; Accepted May 28, 2019

DOI: $10.3892 / \mathrm{mco} .2019 .1871$

\begin{abstract}
Pseudoprogression is not frequently observed in patients with non-small cell lung cancer (NSCLC) who are treated with immune checkpoint inhibitors. We report on a case of pseudoprogression, which was presented as intestinal perforation after pembrolizumab immunotherapy for NSCLC. A-54-year-old man with stage IV NSCLC received pembrolizumab therapy. The patient was admitted to our hospital because of acute abdominal pain and the computed tomography scan revealed diffuse wall thickening of the small bowel with free intraperitoneal air. Intestinal perforation was suspected and surgical resection was performed. Histological evaluation of the resected specimen showed infiltrated lymphocytes positive for CD3, CD8 with necrotic tumor cells, suggestive of an immune reaction. Although intestinal perforation after treatment with immune checkpoint inhibitors is rare, it can be an unusual presentation of pseudoprogression and clinicians should be aware of this possibility.
\end{abstract}

\section{Introduction}

Immune checkpoint inhibitor in non-small cell lung cancer (NSCLC) has been a breakthrough in the treatment of metastatic NSCLC. Among antibodies against two classes of immune checkpoints, anti-PD-1 or anti-PD-L1, nivolumab, and pembrolizumab have been established as novel and promising treatment of NSCLC (1-3). The US FDA approved pembrolizumab as a first-line therapy in October 2016. Since tumor size

Correspondence to: $\mathrm{Dr} \mathrm{Ki}$ Hyeong Lee, Department of Internal Medicine, College of Medicine, Chungbuk National University, 776 Sunhwan-ro 1, Seowon-gu, Cheongju, North Chungcheong 28644, Republic of Korea

E-mail: kihlee@chungbuk.ac.kr

Key words: pseudoprogression, non-small cell lung cancer, intestinal perforation, immunotherapy, pembrolizumab can increase initially due to infiltration of immune cells such as cytotoxic $\mathrm{T}$ lymphocytes surrounding tumors before significant shrinkage of the tumor with immunotherapy, several studies reported pseudoprogression in patients with different types of solid tumors treated with immune checkpoint inhibitors (4-6).

Pseudoprogression is unique response pattern which is observed in patients who are treated with immunotherapeutic agents (7). The initial progression due to infiltration of immune cells such as cytotoxic T lymphocytes surrounding tumors eventually regress. The prevalence of pseudoprogression is quite high in malignant melanoma, but pseudoprogression in NSCLC is not frequently observed $(8,9)$. In addition, intestinal perforation related with pseudoprogression has not been reported yet. In this case, we firstly described the case of pseudoprogression in NSCLC treated with pembrolizumab which was presented as intestinal perforation.

\section{Case report}

A 54-year-old man presented with acute periumbilical pain in February 2018. He had been diagnosed with metastatic NSCLC with adrenal metastasis in August 2017 at Chungbuk National University Hospital. The patient had received second-line therapy with pembrolizumab (200 mg every 3 weeks) since October 2017 after failing first-line therapy with pemetrexed and cisplatin chemotherapy. The abdominal computed tomography (CT) scan showed diffuse enhancing wall thickening at the duodenum through the proximal jejunum and signs of free air in the peritoneal cavity suggesting intestinal perforation (Fig. 1B). The patient underwent emergency laparotomy with segmental resection and anastomosis of the perforated small bowel. Microscopic examination of the resected specimen revealed diffuse transmural involvement of the tumor and massive infiltration of inflammatory cells (Fig. 2A). Immunohistochemistry (IHC) analysis indicated that the infiltrating cells primarily consisted of lymphocytes, especially CD8-positive cells (Fig. 2B and $\mathrm{C}$ ). Histopathological findings including IHC staining for TTF-1, CK7, and CK20 for tumor cells were the same as those of the primary lung mass (data not shown). Formalin-fixed, paraffin embedded (FFPE) tissue were cut using a microtome 
A
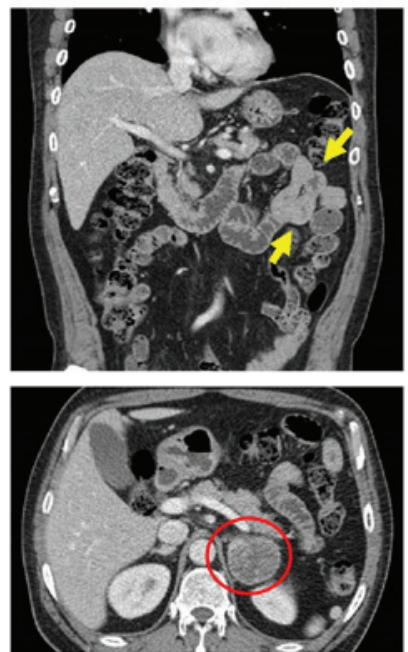
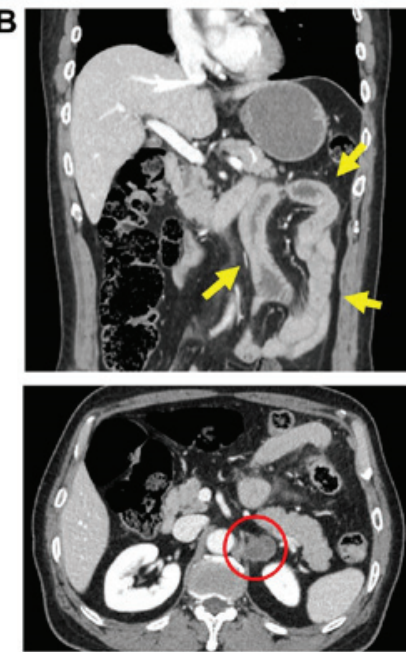
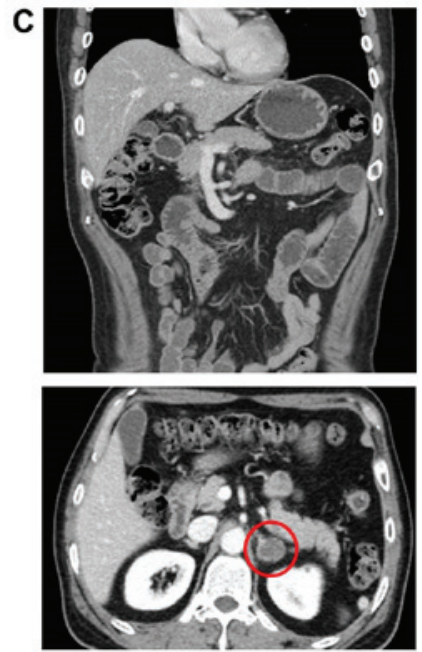

Figure 1. Serial abdominal CT images showing pseudoprogression of the intestinal lesion and corresponding time point responses in the target lesion (left adrenal gland, red circle). (A) At baseline, suspicious focal wall thickening in the proximal jejunum is noted (yellow arrows). (B) After 15 weeks of treatment, the CT scan shows diffuse wall thickening with enhancement in the proximal small intestine (yellow arrows). The left adrenal gland shows a PR (red circle). (C) The intestinal wall thickening spontaneously resolved at 3 weeks after surgery. The left adrenal gland was slightly more decreased (red circle). PR, partial response; CT, computed tomography.

A

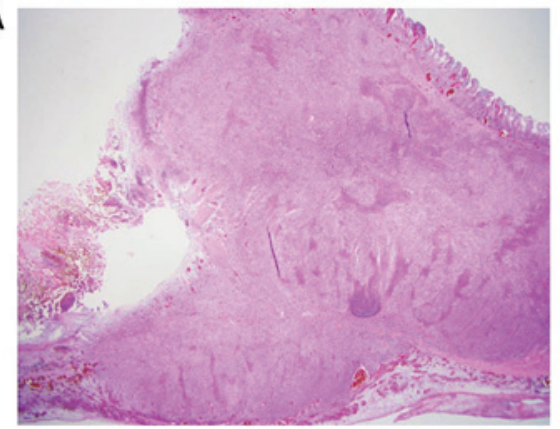

C

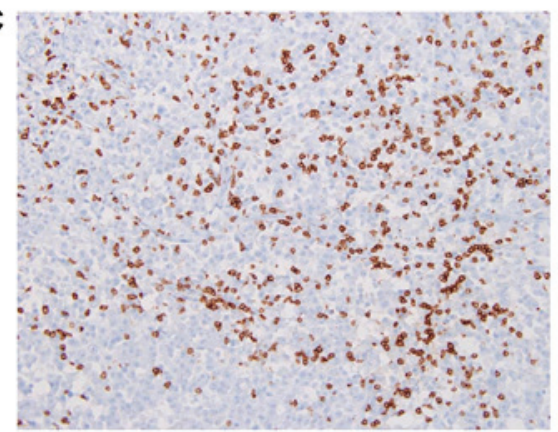

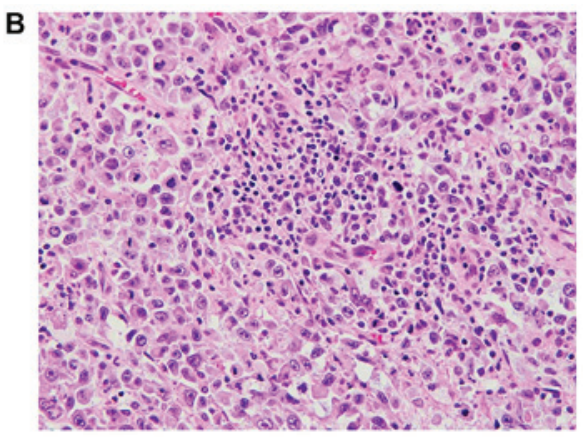

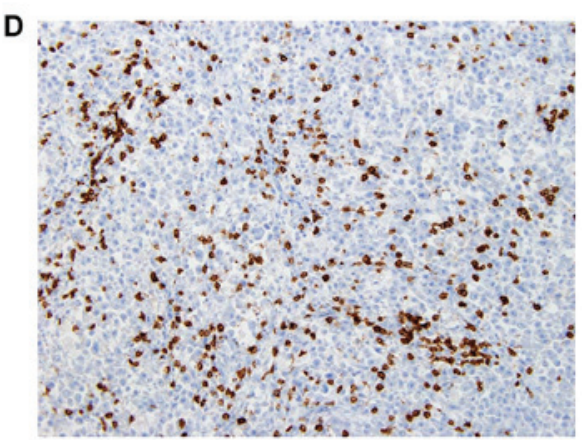

Figure 2. Histopathological findings and immunostaining of surgical specimens. (A) Transmural spread by tumor cells with geographic necrosis (hematoxylin and eosin; original magnification, x12). (B) Poorly differentiated carcinoma with inflammatory cell infiltration (hematoxylin and eosin; original magnification, $x$ 400). (C) Immunohistochemistry shows infiltration of cytotoxic T lymphocytes positive for CD3. Magnification, x200. (D) Immunohistochemistry shows infiltration of cytotoxic T lymphocytes positive for CD8. Magnification, x200.

(RM 2245; Leica Biosystems, Nussloch, Germany) and stained with hematoxylin and eosin (H\&E). IHC staining methods were used according to the manufacturer's protocols; LCA (X16/99, 1:400; Novocastra), CD8 (1A5, 1:50; Cell Marque), TTF-1 (SPT24, 1:250; Cell Marque), CK7 (OV-TL 12/3-0, 1:100; Cell Marque), and CK20 (KS20.8, 1:100; Novocastra) using Benchmark XT autostainer (Ventana). The slides were counterstained with Harris haematoxylin. Retrospective review of the baseline CT scan revealed a suspicious focal wall thickening in the proximal jejunum that was missed at baseline (Fig. 1A).
The primary lung tumor and a metastatic adrenal mass achieved partial response (PR) after two cycles of treatment and remained in PR state at the time of presentation (Fig. 1B). Tumor-related or gastrointestinal symptoms suggestive of intestinal pathology did not develop until the occurrence of the perforation. Treatment was skipped during the postoperative period, and a follow-up CT scan at 3 weeks after surgery showed spontaneous resolution of the small bowel wall thickening (Fig. 1C). The patient recovered without events, and pembrolizumab treatment was resumed 4 weeks after surgery. 


\section{Discussion}

Pseudoprogression is observed in 6.7 to $10 \%$ of patients with melanoma treated with immune checkpoint inhibitors; however, the frequency of pseudoprogression in other solid tumors including NSCLC is below 5\% (8). Although the mechanism of pseudoprogression is not completely understood, it is thought to be caused by infiltration of inflammatory cells leading to transient enlargement of existing tumor masses or appearance of new lesions that eventually regress (10). The pathologic findings in the present case revealed extensive infiltration of immune cells, including cytotoxic CD8-positive T cells, into tumor tissue, which is in line with the immune infiltration theory. However, because tumor volume is largely determined by tumor cells, as shown in this case, further studies are needed to elucidate the mechanism by which the cancer cells transiently proliferate during the course of immunotherapy. Novel response criteria developed for the response evaluation of immunotherapy require confirmation of progressive disease to prevent inappropriate discontinuation of effective therapy $(11,12)$. Discriminating pseudoprogression from true progression is often challenging in clinical practice. In general, patients with pseudoprogression tend to be clinically stable without the aggravation of tumor-related symptoms (11). In the present case, the patient had no gastrointestinal symptoms such as vomiting or diarrhea until the occurrence of the intestinal perforation and rapidly recovered after the operation. There was the possibility that anti-tumor effect of pembrolizumab resulted in intestinal perforation rather than pseudoprogression, the diffuse wall thickening of the small bowel after pembrolizumab (Fig. 1B) in current case remains to be explained. Any tumor marker was not analyzed in the patients, tumor markers could be measured for future cases.

Although pseudoprogression is not frequent in NSCLC, clinicians should be aware of a possible unusual presentation during immunotherapy.

\section{Acknowledgements}

Not applicable.

\section{Funding}

No funding was received.

\section{Availability of data and materials}

All data generated during the present study are available from the corresponding author on reasonable request.

\section{Authors' contributions}

HKK, HSH, CGW and OJL acquired, analyzed and interpreted the data, and drafted the manuscript. SWB, YJ, YY, JK, JYA and TGL interpreted the data and drafted the manuscript. KHL contributed to the conception and design of the study, analyzed and interpreted the data, and drafted the manuscript. All authors read and approved the final manuscript.

\section{Ethics approval and consent to participate}

Not applicable.

\section{Patient consent for publication}

The patient provided written informed consent to publish his data.

\section{Competing interests}

KL has been personally compensated for participating in advisory boards for Boehringer Ingelheim, AstraZeneca and Eli Lilly and Company. The other authors declare that they have no competing interests.

\section{References}

1. Smit EF and Baas P: Lung cancer in 2015: Bypassing checkpoints, overcoming resistance, and honing in on new targets. Nat Rev Clin Oncol 13: 75-76, 2016.

2. Brahmer J, Reckamp KL, Baas P, Crinò L, Eberhardt WE, Poddubskaya E, Antonia S, Pluzanski A, Vokes EE, Holgado E, et al: Nivolumab versus docetaxel in advanced squamous-cell non-small-cell lung cancer. N Engl J Med 373: 123-135, 2015.

3. Garon EB, Rizvi NA, Hui R, Leighl N, Balmanoukian AS, Eder JP, Patnaik A, Aggarwal C, Gubens M, Horn L, et al: Pembrolizumab for the treatment of non-small-cell lung cancer. N Engl J Med 372: 2018-2028, 2015.

4. Hamid O, Robert C, Daud A, Hodi FS, Hwu WJ, Kefford R, Wolchok JD, Hersey P, Joseph RW, Weber JS, et al: Safety and tumor responses with lambrolizumab (anti-PD-1) in melanoma. N Engl J Med 369: 134-144, 2013.

5. Robert C, Ribas A, Wolchok JD, Hodi FS, Hamid O, Kefford R, Weber JS, Joshua AM, Hwu WJ, Gangadhar TC, et al: Anti-p rogrammed-death-receptor-1 treatment with pembrolizumab in ipilimumab-refractory advanced melanoma: A randomised dose-comparison cohort of a phase 1 trial. Lancet 384: 1109-1117, 2014.

6. Motzer RJ, Rini BI, McDermott DF, Redman BG, Kuzel TM, Harrison MR, Vaishampayan UN, Drabkin HA, George S, Logan TF, et al: Nivolumab for metastatic renal cell carcinoma: Results of a randomized phase II trial. J Clin Oncol 33: 1430-1437, 2015.

7. Di Giacomo AM, Danielli R, Guidoboni M, Calabrò L, Carlucci D, Miracco C, Volterrani L, Mazzei MA, Biagioli M, Altomonte $\mathrm{M}$ and Maio M: Therapeutic efficacy of ipilimumab, an anti-CTLA-4 monoclonal antibody, in patients with metastatic melanoma unresponsive to prior systemic treatments: Clinical and immunological evidence from three patient cases. Cancer Immunol Immunother 58: 1297-1306, 2009.

8. Chiou VL and Burotto M: Pseudoprogression and immune-related response in solid tumors. J Clin Oncol 33: 3541-3543, 2015.

9. Kim HK, Heo MH, Lee HS, Sun JM, Lee SH, Ahn JS, Park K and Ahn MJ: Comparison of RECIST to immune-related response criteria in patients with non-small cell lung cancer treated with immune-checkpoint inhibitors. Cancer Chemother Pharmacol 80: 591-598, 2017.

10. Ribas A, Chmielowski B and Glaspy JA: Do we need a different set of response assessment criteria for tumor immunotherapy? Clin Cancer Res 15: 7116-7118, 2009.

11. Seymour L, Bogaerts J, Perrone A, Ford R, Schwartz LH, Mandrekar S, Lin NU, Litière S, Dancey J, Chen A, et al: iRECIST: Guidelines for response criteria for use in trials testing immunotherapeutics. Lancet Oncol 18: e143-e152, 2017.

12. Wolchok JD, Hoos A, O'Day S, Weber JS, Hamid O, Lebbé C, Maio M, Binder M, Bohnsack O, Nichol G, et al: Guidelines for the evaluation of immune therapy activity in solid tumors: Immune-related response criteria. Clin Cancer Res 15: 7412-7420, 2009. 